\title{
Role of a GenoType MTBDRplus line probe assay in early detection of multidrug-resistant tuberculosis at a Brazilian reference center
}

\author{
C.S. Feliciano ${ }^{1}$, M.M.P. Nascimento ${ }^{1}$, L.M.P. Anselmo ${ }^{1}$, R.H.C. Pocente ${ }^{1}$, \\ F. Bellissimo-Rodrigues ${ }^{2}$ and V.R. Bollela ${ }^{1}$ \\ ${ }^{1}$ Departamento de Clínica Médica, Faculdade de Medicina de Ribeirão Preto, Universidade de São Paulo, \\ Ribeirão Preto, SP, Brasil \\ ${ }^{2}$ Departamento de Medicina Social, Faculdade de Medicina de Ribeirão Preto, Universidade de São Paulo, \\ Ribeirão Preto, SP, Brasil
}

\begin{abstract}
Resistance to Mycobacterium tuberculosis is a reality worldwide, and its diagnosis continues to be difficult and time consuming. To face this challenge, the World Health Organization has recommended the use of rapid molecular tests. We evaluated the routine use (once a week) of a line probe assay (Genotype MTBDRplus) for early diagnosis of resistance and for assessment of the main related risk factors over 2 years. A total of 170 samples were tested: 15 (8.8\%) were resistant, and multidrug resistance was detected in 10 (5.9\%). The sensitivity profile took 3 weeks (2 weeks for culture and 1 week for rapid testing). Previous treatment for tuberculosis and the persistence of positive acid-fast smears after 4 months of supervised treatment were the major risk factors observed. The use of molecular tests enabled early diagnosis of drug-resistant bacilli and led to appropriate treatment of the disease. This information has the potential to interrupt the transmission chain of resistant M. tuberculosis.
\end{abstract}

Key words: Multidrug-resistant tuberculosis; Molecular diagnostic techniques; Risk factors

\section{Introduction}

Brazil is among 22 countries that have a concentration of approximately $80 \%$ of all tuberculosis (TB) patients, and occupies the 16th position in absolute numbers of cases (1). Similar to other countries, there has been great progress in controlling the disease in Brazil in the last two decades, since TB was declared to be a worldwide public health emergency by the World Health Organization (WHO) $(1,2)$.

Although there have been consistent advances in controlling measures, there is concern about increasing resistance by Mycobacterium tuberculosis to anti-TB drugs $(1,3)$. The bacillary resistance is still rarely diagnosed, mainly due to the low access to sensitivity tests in countries with greater incidences of the disease $(1,4)$. This is related to the scarcity of available data on the epidemiology of resistance, as evidenced in Brazil $(3,5)$.

In this regard, the development and application of strategies for allowing a fast and effective diagnosis of these cases have gained international attention $(6,7)$, and since 2008, rapid molecular tests for the detection of resistance to anti-TB drugs have been recommended by WHO (8). Among them is the Genotype MTBDRplus (Hain Lifescience, Germany) line probe assay (LPA), approved for use with specimens growing in culture media and also for use with smear-positive sputum samples. This test identifies $M$. tuberculosis complex bacilli and detects mutations in three genes: $r p o B$, which confers resistance to rifampicin; KatG, which confers high-level resistance to isoniazid; and the inhA regulatory region, which confers low-level resistance to isoniazid (8).

The objective of this study was to evaluate the systematic use of an LPA to diagnose resistance of $M$. tuberculosis and the potential meaning of this information in decision making for patients treated in a tertiary reference center in the State of São Paulo, Brazil. We also evaluated possible risk factors associated with bacillary resistance in these patients.

Correspondence: V.R. Bollela, <vbollela@gmail.com>.

Received October 20, 2014. Accepted March 12, 2015. First published online June 30, 2015. 


\section{Material and Methods}

\section{Study population}

All patients at the Hospital das Clínicas, Faculdade de Medicina de Ribeirão Preto, Universidade de São Paulo (USP), who had been diagnosed with tuberculosis by bacillary growth in culture media during 2012 and 2013, were eligible for this study. The project was approved by the Ethics and Research Committee of the Hospital das Clínicas de Ribeirão Preto, USP.

\section{Data collection}

Collecting samples. Clinical samples were collected during routine diagnostic investigation of TB, according to the indication of the medical team that cared for the patient, following the routine already established in the mycobacteria laboratory: direct exam after Ziehl-Neelsen staining and incubation in liquid medium culture in the automated system MGIT 960. Identification of the species was performed by a polymerase chain reaction technique that amplifies a fragment of 123 base pairs of the IS6110 region of $M$. tuberculosis (9).

LPA. The rapid molecular test Genotype MTBDRplus was carried out once a week for all strains isolated in culture during that period, following the manufacturer's instructions (Hain Lifescience). It is a genotypic test that identifies the $M$. tuberculosis complex and detects mutations that confer resistance to rifampicin and isoniazid. The identification of resistance to rifampicin was determined by detection of mutations in the $r p o B$ gene, which codifies the RNA polymerase $\beta$-subunit. With regard to resistance to isoniazid, we evaluated mutations in the KatG gene (that codifies catalase peroxidase), which confers high-level resistance to isoniazid, and in the inhA regulatory region (that codifies the enoyl-acyl carrier protein reductase), which confers low-level resistance to isoniazid (8).

The procedure involved extraction, multiplex amplification with biotinylated primers, and DNA reverse hybridization. The result was determined and interpreted on a strip. The positive control uses a probe, which identifies $M$. tuberculosis complex, marked as TUB in the strip. As quality controls, the strip had the following probes: conjugate control (CC), amplification control (AC), and locus control (rpoB, KatG, and inhA). The strip was also composed of wild-type (WT) probes that included the most important resistance areas of the referred genes (rpoB WT 1 to 8, KatG WT, and inhA WT 1 and 2). If they were present, they excluded detectable mutations in both genes and in the regulatory region that was being evaluated. Other components of the strip were mutation probes, which detected some of the most common mutations that cause resistance. Probes that were positive in the locus of a mutation reflected the mutation in the gene or in the regulatory region evaluated. For the $r p o B$ gene, we evaluated the mutations D516V, H526Y, H526D, and S531L. For the KatG gene, we evaluated mutations S315T1 and $\mathrm{S} 315 \mathrm{~T} 2$. In relation to the inhA regulatory region, we evaluated mutations C15T, A16G, T8C, and T8A. The absence of a WT band or the presence of a mutant band was an indication of resistance to the drug evaluated. For interpretation, the strip was compared with an evaluation form provided by the manufacturer $(8,10)$.

Phenotypic sensitivity testing. Samples that were resistant to rifampicin and/or isoniazid in the LPA were evaluated by the reference laboratory for the resistance of mycobacteria through nonradiometric phenotypic sensitivity testing in liquid medium BACTEC MGIT 960 (MGIT 960; Becton Dickinson Diagnostic Systems, USA). The reference laboratory (which is in the capital of São Paulo state) receives and tests only strains from patients suspected of having resistant TB. The time between sending the strains and receiving the resistance results was 6 weeks.

Risk factors for resistance. Information on risk factors for resistance was obtained through the review of medical records. We evaluated sociodemographic data, habits (elitism, smoking, and drug addiction), history of treatment, previous therapeutic failure, history of contact with cases of multidrug-resistant tuberculosis (MDR-TB), street population, penitentiary population, infection by human immunodeficiency virus (HIV), and alterations in pulmonary imaging examinations.

\section{Statistical analysis}

Data were analyzed by the Stata statistical software, version 12.0 (StataCorp LP, USA). The study involved the description of the sensitivity profile of $M$. tuberculosis strains. To test the association among the variables studied and the occurrence of resistant tuberculosis (to rifampicin, isoniazid, or both), a two-tailed Fisher's exact test was used.

With the aim of determining which of these variables exhibited real association with the outcome of resistant tuberculosis, a model of logistic regression was built, using as independent variables those showing $P \leqslant 0.10$ in univariate analysis using Fisher's test.

\section{Results}

\section{Sensitivity profile and patient characterization}

Samples from 170 patients diagnosed with TB were included in the period of the study (134 men and 36 women; mean age 41.8 years). The pulmonary form of the disease occurred in 120 patients $(70.6 \%)$ and the other 50 patients showed disseminated and/or extrapulmonary forms, with the most frequent site being pleura in 15 cases $(8.8 \%$ of the total).

From the 170 included samples, $155(91.2 \%)$ showed sensitivity to rifampicin and isoniazid, and $15(8.8 \%)$ showed 
some resistance profile. Multidrug-resistant isolates reached $5.9 \%(10 / 170)$ of total cases, monoresistance to rifampicin $1.2 \%(2 / 170)$, and monoresistance to isoniazid $1.7 \%(3 / 170)$.

Primary MDR-TB (patients without prior TB treatment) occurred in two cases (1.2\%) and acquired MDR-TB in eight cases $(4.7 \%)$. Primary monoresistance to isoniazid was detected in one patient $(0.6 \%)$ and acquired was detected in two patients (1.2\%). Primary monoresistance to rifampicin did not occur in this sample, and the acquired resistance to this drug was detected in two patients $(1.2 \%)$.

From 12 samples that exhibited resistance to rifampicin by the molecular test Genotype MTBDRplus, 9 $(75 \%)$ showed the same genotypic profile of resistance, i.e., loss of the wild-type band WT8 and appearance of the MUT3 band of the $r p o B$ gene, which infers the mutation S531L. The genotypic profile of resistance to isoniazid appeared to be very heterogeneous in this sample: five isolates had a high-level resistance profile to the drug (loss of wild-type band and appearance of the band referring to MUT1 of the KatG gene, which infers the mutation S315T1); and the other five isolates showed a low-level resistance pattern. Of those, one showed a loss of the WT2 wild-type band and four showed a loss of WT1 and presence of the band referring to MUT1 of the inhA regulatory region, which infers the mutation C15T (Figure 1).

The mean time for obtaining resistance results of the molecular test was 3 weeks, including the 2 weeks until signaling growth in the liquid medium MGIT-96 and 1 week for the Genotype MTBDRplus sensitivity test.

For those samples with both LPA and reference laboratory multidrug-resistant results, there was the same resistance profile $(100 \%)$ for rifampicin and $80 \%$ for isoniazid. In two of these isolates, the LPA did not demonstrate the resistance detected in the phenotypic testing.

\section{Evaluation of possible risk factors associated with resistance}

Among the possible risk factors analyzed, we determined the association of the grouped variables: brown and black color, history of treatment, and previous therapeutic failure, with statistically significant differences.

The history of contact with a MDR-TB carrier seems to have an association with the development of resistance; however, in the present sample only two patients had this risk factor and there was no statistically significant difference. We demonstrated an association between resistant $\mathrm{TB}$ and the presence of radiological or tomographic findings of pulmonary fibrosis, with statistically significant differences. The presence of pulmonary caves also demonstrated this association, but without any statistically significant difference (Table 1).

With the logistic regression model, we found that only the variable "therapeutic failure" exhibited a real association with the outcome of resistant disease, with an odds ratio of 103.53 and confidence interval between 9.6 and 1109.5 (Table 2). It is important to point out that there was no positive association in patients with HIV/ acquired immune deficiency syndrome (AIDS) in the present study.

\section{Follow-up information for the 15 patients with resistant $M$. tuberculosis}

From 15 patients with confirmed resistance, $11(73.3 \%)$ were cured and $4(26.7 \%)$ died during the treatment. Among the patients who died, three were also infected with HIVIAIDS (1 MDR-TB, 1 rifampicin resistant, and 1 isoniazid resistant). The fourth patient had previous

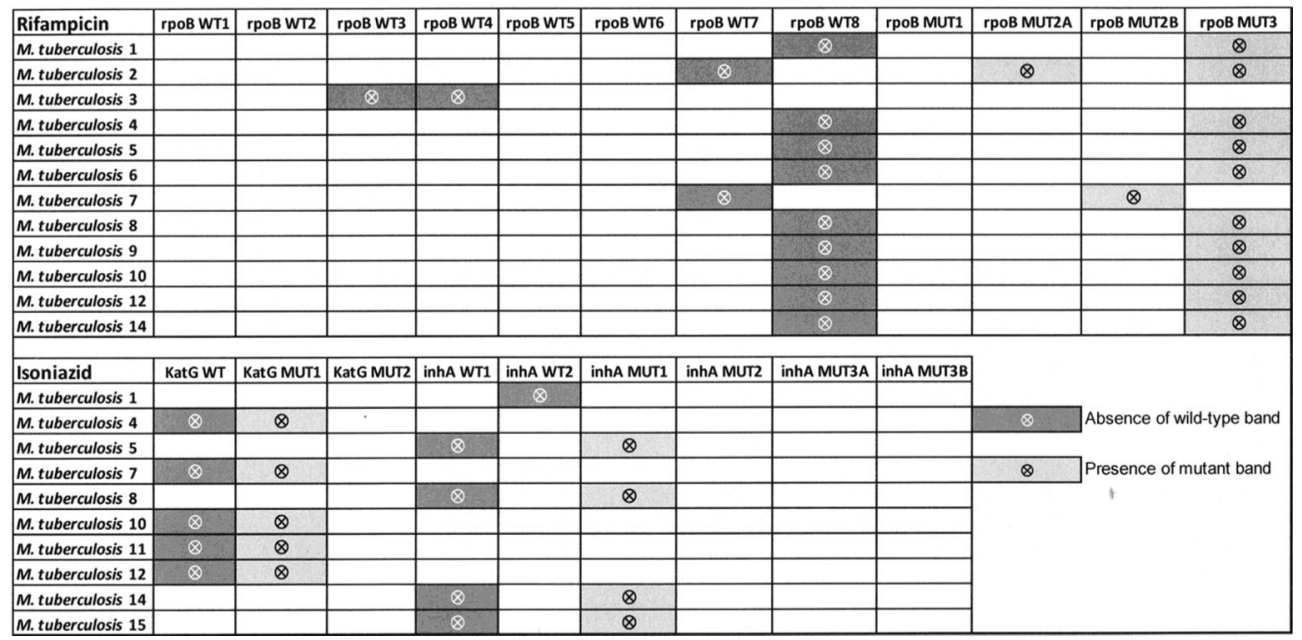

Figure 1. Schematic representation of the Genotype MTBDRplus results in isolates resistant to rifampicin and/or isoniazid. 
Table 1. Analysis of the association between variables studied and the development of resistance to tuberculostatic drugs (rifampicin, isoniazid or both).

\begin{tabular}{|c|c|c|c|}
\hline Risk factors & Sensitive TB & Resistant TB & $P$ \\
\hline Gender & & & 0.198 \\
\hline Male & $120(77.42)$ & $14(93.33)$ & \\
\hline Female & $35(22.58)$ & $1(6.67)$ & \\
\hline Color & & & 0.029 \\
\hline White & $98(63.23)$ & $5(33.33)$ & \\
\hline Brown and black & $57(36.77)$ & $10(66.67)$ & \\
\hline Elitism & & & 0.123 \\
\hline Yes & $116(74.84)$ & $8(53.33)$ & \\
\hline No & $39(25.16)$ & $7(46.67)$ & \\
\hline Smoking & & & 0.042 \\
\hline Yes & $113(72.90)$ & $7(46.67)$ & \\
\hline No & $42(27.10)$ & $8(53.33)$ & \\
\hline Drug addiction & & & 0.059 \\
\hline Yes & $72(46.45)$ & $3(20)$ & \\
\hline No & $83(53.33)$ & $12(80)$ & \\
\hline Previous treatment & & & 0.001 \\
\hline Yes & $29(18.71)$ & $9(60)$ & \\
\hline No & $126(81.29)$ & $6(40)$ & \\
\hline Therapeutic failure & & & $<0.001$ \\
\hline Yes & $1(0.65)$ & $10(66.67)$ & \\
\hline No & $154(99.35)$ & $5(33.33)$ & \\
\hline Contact with MDR-TB & & & 0.169 \\
\hline Yes & $1(0.65)$ & $1(6.67)$ & \\
\hline No & $154(99.35)$ & $14(93.33)$ & \\
\hline Health professional & & & 1.000 \\
\hline Yes & $4(2.58)$ & 0 & \\
\hline No & $151(97.42)$ & $15(100)$ & \\
\hline Street population & & & 1.000 \\
\hline Yes & $12(7.74)$ & 0 & \\
\hline No & $143(92.26)$ & $15(100)$ & \\
\hline Penitentiary population & & & 1.000 \\
\hline Yes & $18(11.61)$ & $1(6.67)$ & \\
\hline No & $137(88.39)$ & $14(93.33)$ & \\
\hline HIVIAIDS & & & 0.400 \\
\hline Yes & $60(42.55)$ & $4(28.57)$ & \\
\hline No & $81(57.45)$ & $10(71.43) a$ & \\
\hline Caves & & & 0.258 \\
\hline Yes & $49(31.61)$ & $7(46.67)$ & \\
\hline No & $106(68.39)$ & $8(53.33)$ & \\
\hline Fibrosis & & & 0.001 \\
\hline Yes & $10(6.45)$ & $6(40)$ & \\
\hline No & $145(93.55)$ & $9(60)$ & \\
\hline
\end{tabular}

Data are reported as number with percent in parenthesis. a: HIV status was not available for one patient. The model of logistic regression was built in univariate analysis using Fisher's test.

MDR-TB, treated and cured in 2009/2010 with a relapse in 2011, when $M$. tuberculosis was isolated again and presented with resistance to rifampicin, isoniazid, and ethambutol, without HIV infection.

\section{Discussion}

Of the 170 strains of $M$. tuberculosis analyzed, 155 $(91.2 \%)$ showed sensitivity both to rifampicin and to isoniazid and $15(8.8 \%)$ showed a resistance profile to both drugs. By evaluating the resistance data in the total sampling, we observed $5.9 \%$ (10 cases) with multidrug resistance, $1.2 \%$ (2 cases) of monoresistance to rifampicin, and $1.7 \%$ (3 cases) of monoresistance to isoniazid. Primary MDR-TB occurred in two $(1.2 \%)$ cases and acquired MDR-TB in eight $(4.7 \%)$ cases.

Data are scarce globally and nationally about resistance to the first line of anti-tuberculosis drugs. This fact used to be related to the scarcity of trained personnel and equipped laboratories to conduct sensitivity tests and poor logistics for the shipment of samples that needed to be tested. These factors have hindered, in practice, more regular analyses of the sensitivity profile of $M$. tuberculosis, especially in countries with a high prevalence of TB, MDR-TB, and tuberculosis and HIV co-infection $(1,3)$.

Data from WHO, between 1994 and 2010, estimated the prevalence of primary and acquired MDR-TB as $3.4 \%$ and $19.8 \%$, respectively (11). It is estimated that, in the year 2012, the incidence of MDR-TB was $5.2 \%$ and it is increasing (1).

Evaluating data from the II Brazilian Inquiry of Resistance to anti-TB drugs, which was carried out between 2007 and 2008, involving 4421 patients, the rate of primary resistance to rifampicin and isoniazid was $1.4 \%$ and acquired resistance was $7.5 \%$. Primary monoresistance to isoniazid was $6 \%$ and acquired was $15.3 \%$. For rifampicin, primary and acquired resistance were $1.5 \%$ and $8 \%$, respectively $(3)$.

In this study, 9 of the 12 samples that showed resistance to rifampicin in the rapid molecular test had the same genotypic profile of resistance (loss of $r p o B$ WT8 wild-type band and appearance of $r p o B$ MUT3 band), which infers the mutation S531L. Hillemann et al. (12) found similar data in their study, in which the mutation S531L occurred in $73.6 \%$ of the samples evaluated by Genotype MTBDRplus. Vijdea et al. (10) and Yadav et al. (13) found, respectively, $86 \%$ and $72 \%$ of the S531L mutation in strains resistant to rifampicin that were subjected to the same genotypic testing.

Different from that observed in the resistance to rifampicin, the genotypic profile to isoniazid resistance was shown to be very heterogeneous in this sample. There were five isolates with high-level resistance profiles to the drug due to mutation S315T1 (KatG gene) and five with low-level resistance patterns, and four of them were due to mutation $\mathrm{C} 15 \mathrm{~T}$ (inhA regulatory region).

Vijdea et al. (10) evaluated two distinct subgroups in relation to the resistance profile to isoniazid. In one of the subgroups, the S315T1 mutation was observed in all isolates tested, and in the other group the mutation $\mathrm{C} 15 \mathrm{~T}$ was the most frequent $(86 \%)$ among the isolates with 
Table 2. Model of logistical regression of the independent variables that showed association with resistance in univariate analysis.

\begin{tabular}{lccrcr}
\hline & Resistant TB (\%) & Sensitive TB (\%) & Univariate P & OR (95\%Cl\%) & Multivariate P \\
\hline Therapeutic failure & 66.67 & 0.65 & $<0.001$ & $103.5(9.6-1109.6)$ & $<0.001$ \\
Previous treatment & 60 & 18.71 & 0.001 & $4.9(0.9-26.9)$ & 0.067 \\
Brown and black & 66.67 & 36.77 & 0.029 & $2.9(0.6-14.8)$ & 0.198 \\
Smoking & 46.67 & 72.90 & 0.042 & $0.4(0.08-2.1)$ & 0.299 \\
Drug addiction & 20 & 46.45 & 0.059 & $0.2(0.02-1.9)$ & 0.185 \\
\hline
\end{tabular}

low-level resistance to the drug. Lacoma et al. (14) observed that, in isolates with high-level resistance, $80.9 \%$ showed the mutation S315T in the KatG gene.

Without the LPA, the mean time for clinicians to identify the M. tuberculosis sensitivity profile in our hospital is from 8 to 10 weeks, including growth time of the bacillus ( 2 weeks), transportation to the reference laboratory, and phenotypic sensitivity testing (6-8 weeks). In this study with the LPA incorporated in the mycobacteria laboratory routine, it was possible to obtain the results of sensitivity tests to rifampicin and isoniazid 3 weeks after receiving the sample in the hospital laboratory. This information may have a potential positive impact on the decision-making process for TB patients, mainly by reducing the time to begin correct treatment, as described by other studies in areas with high prevalence of MDR-TB $(15,16)$.

A finding to be highlighted was the detection of resistance with higher frequency in patients with smearpositive samples 4 months after regular treatment of the disease (characterizing initial treatment failure). This finding is a known sensitive indicator for resistant cases in clinical practice, and is easily available, because it can be obtained during follow-up of patients in treatment by performing smear examinations on a monthly basis during the use of TB medication.

Among the possible risk factors associated with resistance are previous treatments for tuberculosis, as has been frequently reported by many authors (17-20). In this study, previous treatment followed by relapse of the disease was an important risk factor for the development of resistance. This is corroborated by the presence of the highest frequency of radiological alterations of the type pulmonary "fibrosis" among patients who had been treated previously for tuberculosis and cured.

In our study, we did not observe statistically significant differences between the presence of pulmonary cavities and the occurrence of resistance. However, there are some studies demonstrating this association, because both primary and acquired resistances are phenomena dependent on bacillary load and active multiplication, which is much higher in the presence of cavitary disease $(21,22)$.

The association between brown and black colors and M. tuberculosis resistance seems to be related more to the poor socioeconomic and housing conditions of these population groups in Brazil, as already observed in another study in the country (21).

There is great divergence in the literature about the role played by HIV infection, and many authors have not found this association $(19,21,23,24)$. Certainly, the number of TB-resistant cases included in this study limited evaluation of an association between HIV infection and the development of resistance by the bacillus to the two main drugs of the anti-TB scheme. This limitation is also valid for the evaluation of other risk factors analyzed.

An important limitation of this study is the fact that the molecular sensitivity test was only performed on bacilli that grew in liquid culture medium and were from patients who do not represent all the TB cases diagnosed in the hospital during the period of the study. There was a group of patients (less than 20 registered as TB cases) who did not have culture confirmation. Most were immunosuppressed patients with severe disease, suspected of TB, from whom it was not possible to isolate the bacillus, so empirical treatment was initiated.

Another limitation was the lack of phenotypic testing on the strains that were sensitive using the LPA. The reference laboratory does not perform analyses for patients without any indication for TB-resistant testing. However, when reviewing the cases, we observed clinical and microbiological cures with conventional treatment for the cases with LPA bacillus sensitivity to rifampicin and isoniazid.

Finally, this study was not designed to compare patient outcomes before and after LPA introduction to the Mycobacteria laboratory routine, but it definitely contributed to the early start of MDR treatment in our setting.

In conclusion, despite consistent advances in the control of tuberculosis, the challenge of increasing resistance to anti-TB drugs persists. Considering the limited number of recent national data about the epidemiology of $M$. tuberculosis resistance, it becomes urgent to build an epidemiological profile of the bacillary resistance in the country. To reach this goal it would be critical to optimize and increase access to sensitivity tests, and the LPA could be an effective and affordable option. With deeper knowledge of the epidemiological resistance profile, in the future it could be possible to define groups of 
patients at higher risk to host-resistant strains according to the region in which they live. Still, early diagnosis of resistance has the potential to impact the decision for the

\section{References}

1. WHO (World Health Organization). Global tuberculosis report 2013. Geneva: World Health Organization; 2013.

2. Brazil. Ministry of Health. Boletim epidemiológico 44. O controle da tuberculose no Brasil: avanços, inovações e desafios. http://portalsaude.saude.gov.br/images/pdf/ 2014/maio/29/BE-2014-44--2----Tuberculose.pdf. Accessed January 19, 2014.

3. Kritski AL. [Multidrug-resistant tuberculosis emergence: a renewed challenge]. J Bras Pneumol 2010; 36: 157-158, doi: 10.1590/S1806-37132010000200001.

4. Albert $\mathrm{H}$, Bwanga $F$, Mukkada $S$, Nyesiga $B$, Ademun JP, Lukyamuzi $G$, et al. Rapid screening of MDR-TB using molecular Line Probe Assay is feasible in Uganda. BMC Infect Dis 2010; 10: 41, doi: 10.1186/1471-2334-10-41.

5. Bastos GM, Cezar MC, Mello FC, Conde MB. Prevalence of primary drug resistance in pulmonary tuberculosis patients with no known risk factors for such. J Bras Pneumol 2012; 38: 733-739, doi: 10.1590/S1806-37132012000600008.

6. Keshavjee S, Farmer PE. Tuberculosis, drug resistance, and the history of modern medicine. N Engl J Med 2012; 367: 931-936.

7. Nicolau I, Ling D, Tian L, Lienhardt C, Pai M. Research questions and priorities for tuberculosis: a survey of published systematic reviews and meta-analyses. PLoS One 2012; 7: e42479, doi: 10.1371/journal.pone.0042479.

8. WHO (World Health Organization). Molecular line probe assays for rapid screening of patients at risk of multi-drug resistant tuberculosis (MDR-TB). Policy statement. Geneva: World Health Organization; 2008, http://www.who.int/tb/ features_archive/policy_statement.pdf.

9. Bollela VR, Sato DN, Fonseca BA. McFarland nephelometer as a simple method to estimate the sensitivity of the polymerase chain reaction using Mycobacterium tuberculosis as a research tool. Braz J Med Biol Res 1999; 32: 10731076, doi: 10.1590/S0100-879X1999000900003.

10. Vijdea $R$, Stegger $M$, Sosnovskaja $A$, Andersen $A B$, Thomsen VO, Bang D. Multidrug-resistant tuberculosis: rapid detection of resistance to rifampin and high or low levels of isoniazid in clinical specimens and isolates. Eur J Clin Microbiol Infect Dis 2008; 27: 1079-1086, doi: 10.1007/s10096-008-0548-9.

11. Zignol M, van Gemert W, Falzon D, Sismanidis C, Glaziou P, Floyd $\mathrm{K}$, et al. Surveillance of anti-tuberculosis drug resistance in the world: an updated analysis, 2007-2010. Bull World Health Organ 2012; 90: 111-119D, doi: 10.2471/ BLT.11.092585.

12. Hillemann D, Rusch-Gerdes S, Richter E. Evaluation of the GenoType MTBDRplus assay for rifampin and isoniazid susceptibility testing of Mycobacterium tuberculosis strains and clinical specimens. J Clin Microbiol 2007; 45: 2635-2640.

13. Yadav RN, Singh BK, Sharma SK, Sharma R, Soneja M, Sreenivas V, et al. Comparative evaluation of GenoType moment at which to begin treatment and cure, with a possible positive influence on the transmission chain of resistant bacilli.
MTBDRplus line probe assay with solid culture method in early diagnosis of multidrug resistant tuberculosis (MDR-TB) at a tertiary care centre in India. PLoS One 2013; 8: e72036, doi: 10.1371/journal.pone.0072036.

14. Lacoma A, Garcia-Sierra N, Prat C, Ruiz-Manzano J, Haba L, Roses S, et al. GenoType MTBDRplus assay for molecular detection of rifampin and isoniazid resistance in Mycobacterium tuberculosis strains and clinical samples. J Clin Microbiol 2008; 46: 3660-3667.

15. Kipiani M, Mirtskhulava V, Tukvadze N, Magee M, Blumberg HM, Kempker RR. Significant clinical impact of a rapid molecular diagnostic test (Genotype MTBDRplus assay) to detect multidrug-resistant tuberculosis. Clin Infect Dis 2014; 59: 1559-1566, doi: 10.1093/cid/ciu631.

16. Jacobson KR, Theron D, Kendall EA, Franke MF, Barnard $M$, van Helden PD, et al. Implementation of genotype MTBDRplus reduces time to multidrug-resistant tuberculosis therapy initiation in South Africa. Clin Infect Dis 2013; 56: 503-508, doi: 10.1093/cid/cis920.

17. Faustini A, Hall AJ, Perucci CA. Risk factors for multidrug resistant tuberculosis in Europe: a systematic review. Thorax 2006; 61: 158-163, doi: 10.1136/thx.2005.045963.

18. Liang L, Wu Q, Gao L, Hao Y, Liu C, Xie Y, et al. Factors contributing to the high prevalence of multidrug-resistant tuberculosis: a study from China. Thorax 2012; 67: 632-638, doi: 10.1136/thoraxjnl-2011-200018.

19. Ricks PM, Mavhunga F, Modi S, Indongo R, Zezai A, Lambert LA, et al. Characteristics of multidrug-resistant tuberculosis in Namibia. BMC Infect Dis 2012; 12: 385, doi: 10.1186/1471-2334-12-385.

20. Skrahina A, Hurevich H, Zalutskaya A, Sahalchyk E, Astrauko A, Hoffner S, et al. Multidrug-resistant tuberculosis in Belarus: the size of the problem and associated risk factors. Bull World Health Organ 2013; 91: 36-45, doi: 10.2471/BLT.12.104588.

21. Barroso EC, Mota RMS, Santos RO, Sousa ALO, Barroso JB, Rodrigues JLN. Risk factors for acquired multidrugresistant tuberculosis. J Pneumol 2003; 29: 89-97.

22. Coelho AG, Zamarioli LA, Telles MA, Ferrazoli L, Waldman EA. A study of multidrug-resistant tuberculosis in risk groups in the city of Santos, São Paulo, Brazil. Mem Inst Oswaldo Cruz 2012; 107: 760-766, doi: 10.1590/S0074-027620120 00600009 .

23. Micheletti VC, Moreira JS, Ribeiro MO, Kritski AL, Braga JU. Drug-resistant tuberculosis in subjects included in the Second National Survey on Antituberculosis Drug Resistance in Porto Alegre, Brazil. J Bras Pneumol 2014; 40: 155163, doi: 10.1590/S1806-37132014000200009.

24. Suchindran S, Brouwer ES, Van RA. Is HIV infection a risk factor for multi-drug resistant tuberculosis? A systematic review. PLoS One 2009; 4: e5561, doi: 10.1371/journal. pone.0005561. 